\title{
Akzeptanz und Ablehnung beim Übertritt in die Sekundarstufe I
}

Stephan Rösselet und Markus P. Neuenschwander

\section{Zusammenfassung}

Mit dem Übertritt in die Sekundarstufe I treten die Kinder in leistungssegregierte Schulniveaus ein und müssen ihre soziale Position in der Klasse neu aushandeln. Veränderungen der Beliebtheitsdimensionen Akzeptanz und Ablehnung beim Übertritt wurden bisher kaum untersucht. Mit Daten der Längsschnittstudie Wirkungen der Selektion WiSel wurden Akzeptanz und Ablehnung von Kindern im 5., 6. und 7. Schuljahr mit soziometrischen Verfahren erhoben. Es wurden 191 Kinder aus den Kantonen Aargau und Basel-Landschaft mit Übertritt nach dem 5. Schuljahr und 255 Kinder aus den Kantonen Bern und Luzern mit Übertritt nach dem 6. Schuljahr analysiert. Die Ergebnisse zeigen, dass hohe Akzeptanz am Ende der Primarschule mit guten Leistungen, guten Noten und hohem sozialen Selbstkonzept zusammenhängen. Diese Zusammenhänge treten aber am Anfang der Sekundarstufe I nicht auf. Die Beliebtheit ist beim Übergang in die Sekundarstufe I weniger stabil als beim Verbleib im gleichen Schultyp. Das Schulniveau und die Leistungsposition in der Klasse in Mathematik beeinflussen die Veränderung der Beliebtheit nach dem Übergang. Damit kann erstmals gezeigt werden, wie sich soziale Vergleichsprozesse auf die Veränderung der Beliebtheit beim Übertritt in die Sekundarstufe I auswirken. Schulleistungen und zugewiesenes Schulniveau auf der Sekundarstufe I beeinflussen nicht nur das Selbstkonzept, sondern auch die Beliebtheit der Schülerinnen und Schüler.

\section{Schlagworte}

Soziogramm, Beliebtheit, Transition in die Sekundarstufe I 


\section{$1 \quad$ Einleitung}

Peers sind wichtige Bezugspersonen von Jugendlichen. Sie beeinflussen ihre kognitive und soziale Entwicklung, sind für die Befriedigung des Bedürfnisses nach Kontakt, Nähe und Austausch zentral, wirken unterstützend bei der Bewältigung von Übergängen und Entwicklungsaufgaben und fördern ihre Identitätsentwicklung und Selbstpräsentation (Hannover \& Kessels, 2009). Jugendliche wollen bei Peers beliebt sein, um sich sozial zu integrieren und ihren Selbstwert zu sichern (Neuenschwander \& Hascher, 2003). Ihre Beliebtheit hängt mit schulischen Leistungen und Prüfungsversagen sowie mit ihrer psychosozialen Entwicklung zusammen (z.B. Aggressivität, Verhaltens, motorische und Aufmerksamkeitsprobleme, Kontrollüberzeugungen, Drogenmissbrauch, Delinquenz, vgl. Allen, Porter, McFarland, Marsh \& McElhaney, 2005; Ollendick, Weist, Borden \& Greene, 1992).

Die Gründe für die Unterschiede in der Beliebtheit wurden vielfach untersucht (z. B. Newcomb, Bukowski \& Pattee, 1993). Weit weniger ist darüber bekannt, ob und wie sich die Beliebtheit bei schulischen Übergängen verändert. Mit der vorliegenden Studie wird exemplarisch der Übertritt von der Primarstufe in die Sekundarstufe I untersucht. Dabei stehen Zusammenhänge der Beliebtheit mit schulischen Leistungen im Fokus, weil die Selektion leistungsbasiert ist und weil sie zu neuen, leistungshomogenen Klassen führt. Nach dem Übertritt müssen sich die Schülerinnen und Schüler mit einer veränderten Bezugsgruppe auseinandersetzen, was neue soziale Vergleichsprozesse zur Folge hat. Im vorliegenden Kapitel wird folgenden Fragen nachgegangen:

1. Wie unterscheiden sich Schülerinnen und Schüler, die in ihrer Klasse unterschiedlich beliebt sind, in ihren schulischen Leistungen und ihrem sozialen Selbstkonzept?

2. Wie stabil bleibt die Beliebtheit beim Übertritt in die Sekundarstufe I?

3. Inwiefern sagen schulische Leistungen und zugewiesenes Sekundarstufe I Niveau Veränderungen der Beliebtheit nach dem Übertritt in die Sekundarstufe I vorher?

\section{Zwei Dimensionen der Beliebtheit: Akzeptanz und Ablehnung}

Die mittels soziometrischer Verfahren gemessene Beliebtheit einer Schülerin oder eines Schülers beschreibt, wie sehr sie oder er von den Peers innerhalb des Klassenverbands gemocht wird (Hannover \& Kessels, 2009). Die Beliebtheit einer 
Schülerin oder eines Schülers stellt einen Ausdruck der Gefühle der Peers dar. Diese Gefühle hängen davon $a b$, ob und wie sehr sie die Handlungen und Eigenschaften der beurteilten Person für sie - z. B. in Bezug auf eine enge Freundschaft (Babad, 2001) - als belohnend empfinden (vgl. Thibaut \& Kelley, 1959).

Zur soziometrischen Messung von Beliebtheit nennen die Befragten eine bestimmte Anzahl Personen, die sie am liebsten und am wenigsten mögen oder mit denen sie am liebsten oder am wenigsten gern bestimmte Aktivitäten durchführen würden (liking und disliking nominations, Moreno, 1978; Moreno, 1960). Dies ermöglicht, zwei Dimensionen der Beliebtheit voneinander zu unterscheiden, die unabhängig voneinander analysiert werden können: Akzeptanz und Ablehnung (Rubin, Bukowski \& Parker, 2006). Auch ihre relativ geringe, negative Korrelation ( $r=.39$, Rost \& Czeschlik, 1994) spricht dafür, dass sie zwei unterschiedliche Dimensionen sind und nicht zwei Pole einer Dimension dasrtellen. Die getrennte Analyse der beiden Konzepte erlaubt es, die spezifischen Bedingungen von Akzeptanz und Ablehnung zu analysieren.

\subsection{Validierung von Akzeptanz und Ablehnung}

Empirische Untersuchungen zeigen, dass persönliche Eigenschaften, sozialer Hintergrund und die Beziehung zu den Eltern für die Beliebtheit relevant sind (z. B. Attili, Vermigli \& Roazzi, 2010; Dunkake, 2012; Tamm, Kasearu \& Tulviste, 2014). Bessere Schulleistungen gehen mit höherer Akzeptanz einher, während schulleistungsschwächere Schülerinnen und Schüler auf mehr Ablehnung stossen (Hatzichristou \& Hopf, 1996; Newcomb et al., 1993; Rost \& Czeschlik, 1994; Wentzel, 2005). Leistungsstarke sowie intelligente Schülerinnen und Schüler weisen Eigenschaften auf, die anderen das Erreichen von schulischen Zielen erleichtern: Sie vermitteln schulbezogene Werte und Erwartungen, dienen als positive Modelle für das Lernverhalten und geben nützlichen Rat und praktische Hilfestellungen (Hannover \& Kessels, 2009).

Für die Beliebtheit innerhalb einer Klasse sind die Leistungen und die kognitiven Fähigkeiten im internen Klassenvergleich (relative Leistungen) besonders relevant (vgl. z. B. Dunkake, 2012). Schulleistungen der Mitschülerinnen und Mitschüler sind zum einen direkt im Unterricht beobachtbar. Zum anderen sind sie indirekt über Schulnoten beobachtbar, welche als wichtige Indikatoren für die schulischen Leistungen dienen. Noten sind jedoch kein Abbild objektiver schulischer Leistungsstärke. Sie bilden Rangplätze der Schülerinnen und Schüler innerhalb der Klasse ab. Lehrpersonen greifen bei der Leistungsbeurteilung auf ein klasseninternes Bezugssystem zurück (z. B. Trautwein \& Baeriswyl, 2007), welches für klassenin- 
terne Leistungsvergleiche relevanter sein dürfte als Ergebnisse von Leistungstests. Zusammenfassend formulieren wir folgende Hypothesen:

Hypothese 1: Akzeptierte Schülerinnen und Schüler des 6. Schuljahres weisen im Vergleich mit ihren weniger akzeptierten Klassenkameradinnen und kameraden bessere Schulleistungen (relative Leistungen) und Noten auf.

Hypothese 2: Abgelehnte Schülerinnen und Schüler des 6. Schuljahres weisen im Vergleich mit ihren weniger abgelehnten Klassenkameradinnen und kameraden schlechtere Schulleistungen (relative Leistungen) und Noten auf.

Weiter spielen soziale Kompetenzen wie Kooperationsfähigkeit, Hilfsbereitschaft, Kontaktfreudigkeit, Durchsetzungsfähigkeit, Führungsqualitäten (Hannover \& Kessels, 2009; Newcomb et al., 1993; Wentzel, 2005) sowie Persönlichkeitsmerkmale (van der Linden, Scholte, Cillessen, Nijenhuis \& Segers, 2010) für Akzeptanz und Ablehnung eine wichtige Rolle. Es ist davon auszugehen, dass auch soziale Selbstkonzepte, welche ein Urteil über die eigenen sozialen Kompetenzen darstellen, mit der Beliebtheit zusammenhängen. Zudem stossen Schülerinnen und Schüler, die sich zurückziehen und stärker von Depressionen und Ängstlichkeit betroffen sind, auf Ablehnung (Cillessen, van Ijzendoorn, Van Lieshout \& Hartup, 1992; Parkhurst \& Asher, 1992). Eine positive Lebenseinstellung (Grob et al., 1991) dürfte demgegenüber mit höherer Beliebtheit einhergehen.

Hypothese 3: Akzeptierte Schülerinnen und Schüler des 6. Schuljahres schätzen sich im Vergleich mit ihren weniger akzeptierten Klassenkameradinnen und kameraden als offener, durchsetzungs- und konfliktfähiger ein und weisen eine positivere Lebenseinstellung auf.

Hypothese 4: Abgelehnte Schülerinnen und Schüler des 6. Schuljahres schätzen sich im Vergleich mit ihren weniger abgelehnten Klassenkameradinnen und kameraden als weniger offen, durchsetzungs- und konfliktfähig ein und weisen eine weniger positive Lebenseinstellung auf. 


\section{Stabilität und Veränderung der Beliebtheit beim Übertritt in die Sekundarstufe I}

Akzeptanz und Ablehnung von Schülerinnen und Schülern sind über die Zeit relativ stabil. Dies gilt sowohl für Kinder in der Vorschule (Walker, 2009) als auch für Jugendliche in der Oberstufe (Lansford, Killeya Jones, Miller \& Costanzo, 2009). Studien zeigten, dass die Stabilität mit zunehmendem Alter der Schülerinnen und Schüler sogar noch zunimmt (Jiang \& Cillessen, 2005). Weil beim Übertritt von der Primarstufe in die Sekundarstufe I neue Klassen gebildet werden, wichtige Bezugspersonen wegfallen und bestehende Hierarchien unter den Schülerinnen und Schülern verloren gehen, sind bei Schulübertritten geringere Stabilitäten zu erwarten (Jiang \& Cillessen, 2005; Pellegrini \& Bartini, 2000). Dies gilt weniger stark für die Ablehnung als für die Akzeptanz (Hardy, Bukowski \& Sippola, 2002).

Hypothese 5: Akzeptanz und Ablehnung sind beim Verbleib in der gleichen Klasse über die Zeit stabil.

Hypothese 6: Akzeptanz und Ablehnung sind bei einem Klassenwechsel in Folge des Übertritts von der Primarstufe in die Sekundarstufe I über die Zeit weniger stabil als beim Verbleib in der gleichen Klasse (vgl. Hypothese 5).

\section{Determinanten der Veränderung beim Übertritt in die Sekundarstufe I}

Beim Übertritt in die Sekundarstufe I wechseln Schülerinnen und Schüler in leistungssegregierte Schulniveaus. Dadurch werden die Leistungen innerhalb der Klassen homogener, während sie sich zwischen den Schulniveaus stärker unterscheiden. Wie stark die Klassenzusammensetzung die Schülerinnen und Schüler beeinflussen, konnte in Bezug auf das schulische Selbstkonzept mit dem Big-Fish-Little-Pond-Effekt eindrücklich belegt werden (Marsh, 1987, 2005). Ähnliche Referenzgruppeneffekte sind auch bei der Beliebtheit zu erwarten. Wir gehen davon aus, dass analog zur Beurteilung eigener schulischer Fähigkeiten auch die Beliebtheit der Klassenkameradinnen und -kameraden im Klassenverband von den Leistungen der gesamten Klasse abhängen. Wir nehmen deshalb an, dass ein hohes Leistungsniveau der Klasse nach dem Übertritt die Beliebtheit von Schülerinnen und Schülern negativ beeinflusst, weil deren individuelle Leistung im Lichte der leistungsstärkeren Referenzgruppe weniger gut erscheint. Wir erwarten auch, dass 
Schülerinnen und Schüler, die, verglichen mit ihrer neuen Klasse, gute Leistungen (relative Leistungen) erbringen, an Beliebtheit gewinnen. Während sprachliche Kenntnisse nicht nur im Fach Deutsch, sondern auch in anderen Fächern und ausserhalb der Schule gelernt, angewendet und gefördert werden, ist dies für Mathematik kaum der Fall (Angelone, Keller \& Moser, 2013). Wir erwarten deshalb positive Individualeffekte der Leistungen für das Fach Mathematik. Die Leistungen im Fach Deutsch sind aber für die Beliebtheit unerheblich.

Hypothese 7: Die Akzeptanz sinkt beim Übertritt in Klassen mit höheren Anforderungen, während sie beim Übertritt in Klassen mit niedrigeren Anforderungen zunimmt.

Hypothese 8: Die Ablehnung steigt beim Übertritt in Klassen mit höheren Anforderungen, während sie beim Übertritt in Klassen mit niedrigeren Anforderungen sinkt.

Hypothese 9: Die Akzeptanz von Schülerinnen und Schülern, die nach dem Übertritt bessere schulische Leistungen in Mathematik im Vergleich zum Klassendurchschnitt aufweisen (relative Leistungen), nimmt zu. Sie nimmt bei schlechteren relativen Mathematikleistungen ab.

Hypothese 10: Die Ablehnung von Schülerinnen und Schülern, die nach dem Übertritt bessere relative Mathematikleistungen aufweisen, nimmt ab. Sie nimmt bei schlechteren relativen Mathematikleistungen zu.

\section{$5 \quad$ Methode}

Zur Prüfung der formulierten Hypothesen wurden Daten der Schweizer Längsschnittstudie Wirkungen der Selektion ${ }^{1}$ - kurz WiSel - herangezogen, die am Zentrum Lernen und Sozialisation der Pädagogischen Hochschule FHNW durchgeführt wurde.

Schülerinnen und Schüler (5., 6. und 7. Schuljahr) aus vier Deutschschweizer Kantonen füllten in den Herbstsemestern der Schuljahre 2011/2012, 2012/2013 und 2013/2014 schriftliche Fragebögen aus und lösten Leistungstests in den Fächern Mathematik und Deutsch. Die Erhebungen in den verschiedenen Kantonen erlaubten den Vergleich unterschiedlicher Übertrittszeitpunkte in die Sekundarstufe I. Die

1 Wir danken dem Schweizerischen Nationalfonds für die Finanzierung dieser Studie (Projektnummer 100013_134594/1). Wir danken Benno Rottermann und Edith Niederbacher für die Arbeit bei der Durchführung der Studie. 
Schülerinnen und Schüler der Kantone Aargau und Basel-Landschaft traten nach dem 5. Schuljahr von der Primarschule in die Sekundarstufe I über. Diejenigen aus den Kantonen Bern und Luzern traten ein Jahr später, nach dem 6. Schuljahr, über.

\subsection{Stichprobe}

Für die vorliegenden Analysen wurden diejenigen Schülerinnen und Schüler einbezogen, für welche zu allen drei Messzeitpunkten Werte bei den Variablen zur Akzeptanz und Ablehnung vorlagen. Weil diese Variablen auf Urteilen der Mitschülerinnen und Mitschüler basieren und sich die Klassenzusammensetzungen beim Übertritt in die Sekundarstufe I veränderten, wurde auf eine Schätzung der fehlenden Werte verzichtet. Schülerinnen und Schüler in Klassen, in denen zumindest an einem der drei Messzeitpunkte weniger als zehn Schülerinnen und Schüler an den Erhebungen teilgenommen hatten, wurden von den Analysen ausgeschlossen.

Von den verbliebenen 446 Schülerinnen und Schülern gehörten 191 der Gruppe mit Übertritt nach dem 5. Schuljahr und 255 der Gruppe mit Übertritt nach dem 6. Schuljahr an. Mädchen $(231,51.8 \%)$ und Jungen $(215,48.2 \%)$ waren in etwa gleich stark vertreten. 313 (70.2\%) Schülerinnen und Schüler waren Schweizerin oder Schweizer, 92 (20.6\%) Ausländerin oder Ausländer und 41 (9.2\%) Doppelbürgerin oder Doppelbürger. Diese Verhältnisse entsprechen in etwa denjenigen der Schweizer Primarstufe im Schuljahr 2011/2012².

\subsection{Instrumente und Variablen}

Zur Beantwortung der formulierten Hypothesen werden folgende Variablen des Fragebogens und der Leistungstests benutzt ${ }^{3}$ :

2 vgl. https://www.bfs.admin.ch/bfs/de/home/statistiken/bildung-wissenschaft/personen-ausbildung/obligatorische-schule.assetdetail.246173.html

3 Eine ausführliche Beschreibung der eingesetzten Erhebungsinstrumente ist in den Dokumentationsbänden der Studie zu finden (Neuenschwander, Rösselet, Niederbacher, Rottermann \& Scheffler, 2014; Neuenschwander, Rösselet, et al., 2013; Neuenschwander, Rösselet, Scheffler \& Rottermann, 2014; Neuenschwander, Rottermann, Niederbacher, Rösselet \& Scheffler, 2014; Neuenschwander, Rottermann \& Rösselet, 2013; Neuenschwander, Rottermann, Scheffler \& Rösselet, 2014). Download: http://www.fhnw.ch/ $\mathrm{ph} /$ zls/interne-berichte/forschungsberichte/forschungsberichte-wisel. 
1. Beliebtheit (5., 6. und 7. Schuljahr): Akzeptanz und Ablehnung mit je einem Item: „Neben welchen zwei Schülerinnen oder Schülern deiner Klasse möchtest Du am liebsten/am wenigsten gerne sitzen?". Für alle Schülerinnen und Schüler wurde berechnet, wie oft sie gewählt wurden. Damit die Klassengrösse und die Anzahl der Teilnehmenden pro Klasse die Werte nicht verzerrten, wurden die Wahlhäufigkeiten daran relativiert: Der berechnete Wert entspricht einer Schätzung der Prozentzahl der Schülerinnen und Schüler, von denen die Schülerin oder der Schüler gewählt worden wäre, wenn die vollständige Klasse an den Erhebungen teilgenommen hätte. Da diese Werte nicht normalverteilt sind, wurden sie anhand der Auftretenshäufigkeit in Terzile aufgeteilt.

2. Schulnoten Deutsch und Mathematik (7. Schuljahr): Schülerangaben rückblickend auf das Ende des vorangegangenen Schuljahres (1: tiefste Note, 6: höchste Note).

3. Schulniveau Sekundarstufe I (6. / 7. Schuljahr): dreistufig (tiefe, mittlere, hohe Anforderungen).

4. Soziales Selbstkonzept (6. Schuljahr): Drei Faktoren (1) Offenheit (drei Items, z. B. „Einer guten Freundin oder einem guten Freund vertraue ich auch Dinge an, die mir unangenehm sind“, $\alpha=.77$ ), (2) Durchsetzungsvermögen (vier Items, z. B. „Andere Jugendliche hören auf mich“, $\alpha=.67$ ), (3) Konfliktfähigkeit (vier Items, z. B. „Wenn ich eine Meinungsverschiedenheit mit jemandem habe, kann ich sehr gut akzeptieren, dass die oder der Andere nicht die gleiche Meinung hat wie ich ", $\alpha=.67$ ), Antwortskala jeweils von 1 ,stimmt überhaupt nicht', bis 6 ,stimmt voll und ganz:

5. Positive Lebenseinstellung (6. Schuljahr): Acht Items (z. B. „Ich bin zufrieden mit der Art und Weise, wie sich meine Lebenspläne verwirklichen", $\alpha=.86$ ), Antwortskala von 1 ,stimmt überhaupt nicht' bis 6 ,stimmt voll und ganz'.

6. Leistungen und relative Leistungen Deutsch und Mathematik (6. und 7. Schuljahr): Auswahl von Aufgaben von Moser, Buff, Angelone \& Hollenweger (2011) mit einem Ankeritem-Design (Wiederholung einer Teilmenge der Items zu den beiden Messzeitpunkten). Auf der Grundlage der Item Response Theorie (Yen \& Fitzpatrick, 2006) wurde mittels Linking-Methoden (Haberman, 2009) die Leistungen auf einer Metrik abgebildet. Damit können tatsächliche Veränderungen der Kompetenzwerte (Weighted Likelihood Estimates, vgl. Warm, 1989) über mehrere Messzeitpunkte beschrieben werden. Die relativen Leistungen wurden berechnet, indem von der individuellen Leistung der Mittelwert der jeweiligen Klasse abgezogen wurde. 


\subsection{Durchführung der Erhebungen}

Vor den Erhebungen wurde von den Kantonen, den Schulleitungen, den Lehrpersonen und den Eltern das ausdrückliche Einverständnis zur Teilnahme an der Studie eingeholt. Die Teilnahme der Schülerinnen und Schüler war freiwillig. Instruierte Projektmitarbeitende leiteten die Schülerbefragung und die Leistungstests gemäss einer standardisierten Instruktion an. Die teilnehmenden Schülerinnen und Schüler füllten während der Unterrichtszeit im Klassenverband zuerst den Schülerfragebogen und zwei bis drei Wochen später die Leistungstests aus.

\section{$6 \quad$ Ergebnisse}

\subsection{Validierung der Beliebtheit}

Zur Validierung von Akzeptanz und Ablehnung und Testung der Hypothesen 1 bis 4 wurden mehrere einfaktorielle Varianzanalysen durchgeführt. Es wurden die Daten des 6. Schuljahres herangezogen. Damit kann die Validierung der Akzeptanz und der Ablehnung für die Situation am Ende der Primarstufe und zu Beginn der Sekundarstufe I anhand von Daten aus dem gleichen Schuljahr vorgenommen werden. In diesen Analysen sind Akzeptanz und Ablehnung jeweils die unabhängigen Variablen. Die drei Terzile (unteres, mittleres, oberes Terzil) stellen ihre Ausprägungen dar.

Die Hypothesen 1 und 2 werden mit den abhängigen Variablen Testleistungen, relative Testleistungen und Noten geprüft. Zur Prüfung von Hypothese 3 und 4 sind die drei Dimensionen Offenheit, Durchsetzungsvermögen, Konfliktfähigkeit des sozialen Selbstkonzepts und die positive Lebenseinstellung die abhängigen Variablen. 
Tab. 1 Varianzanalysen zur Validierung von Akzeptanz und Ablehnung im 6. Schuljahr

\begin{tabular}{|c|c|c|c|c|c|c|c|c|c|c|c|c|}
\hline & \multicolumn{6}{|c|}{ Akzeptanz Ende Prim. } & \multicolumn{6}{|c|}{ Akzeptanz Anfang Sek I } \\
\hline & I & II & III & $\mathrm{F}$ & & $\mathrm{df}_{1,2}$ & I & II & III & $\mathrm{F}$ & & $\mathrm{df}_{1,2}$ \\
\hline LM & 0.55 & 0.52 & 0.67 & 0.76 & & 2,247 & 0.66 & 0.54 & 0.33 & 1.92 & & 2,185 \\
\hline LD & 0.17 & 0.35 & 0.47 & 1.95 & & 2,246 & 0.57 & 0.28 & 0.03 & 3.96 & * & 2,184 \\
\hline RLM & 0.06 & 0.05 & 0.29 & 2.17 & & 2,247 & -0.03 & -0.02 & -0.19 & 1.66 & & 2,185 \\
\hline RLD & 0.03 & 0.16 & 0.34 & 2.25 & & 2,246 & 0.14 & -0.10 & -0.17 & 2.62 & & 2,184 \\
\hline NM & 4.84 & 4.87 & 5.05 & 2.87 & & 2,248 & 4.58 & 4.62 & 4.54 & 0.24 & & 2,184 \\
\hline ND & 4.86 & 4.99 & 5.19 & 7.30 & ** & 2,246 & 4.66 & 4.69 & 4.81 & 1.80 & & 2,185 \\
\hline $\mathrm{OF}$ & 4.80 & 4.71 & 4.95 & 1.49 & & 2,251 & 4.71 & 5.07 & 5.04 & 2.68 & & 2,188 \\
\hline DV & 4.32 & 4.33 & 4.61 & 4.80 & $* *$ & 2,252 & 4.28 & 4.40 & 4.53 & 1.63 & & 2,188 \\
\hline KF & 4.54 & 4.43 & 4.54 & 0.84 & & 2,252 & 4.29 & 4.47 & 4.30 & 1.25 & & 2,188 \\
\hline \multirow[t]{3}{*}{ PLE } & 4.85 & 4.93 & 5.12 & 4.50 & * & 2,252 & 4.70 & 4.85 & 4.81 & 0.70 & & 2,188 \\
\hline & \multicolumn{6}{|c|}{ Ablehnung Ende Prim. } & \multicolumn{6}{|c|}{ Ablehnung Anfang Sek I } \\
\hline & I & II & III & $\mathrm{F}$ & & $\mathrm{df}_{1,2}$ & I & II & III & $\mathrm{F}$ & & $\mathrm{df}_{1,2}$ \\
\hline LM & 0.64 & 0.62 & 0.41 & 1.49 & & 2,247 & 0.46 & 0.80 & 0.32 & 2.99 & & 2,185 \\
\hline LD & 0.44 & 0.55 & -0.10 & 7.72 & $* *$ & 2,246 & 0.23 & 0.30 & 0.42 & 0.46 & & 2,184 \\
\hline RLM & 0.22 & 0.15 & -0.09 & 2.77 & & 2,247 & -0.10 & -0.02 & -0.08 & 0.29 & & 2,185 \\
\hline RLD & 0.27 & 0.41 & -0.26 & 8.83 & $* *$ & 2,246 & -0.14 & -0.15 & 0.30 & 5.12 & $* *$ & 2,184 \\
\hline NM & 5.04 & 4.78 & 4.75 & 6.28 & $* *$ & 2,248 & 4.58 & 4.70 & 4.45 & 1.62 & & 2,184 \\
\hline ND & 5.12 & 5.03 & 4.75 & 8.89 & $* *$ & 2,246 & 4.76 & 4.69 & 4.63 & 1.30 & & 2,185 \\
\hline OF & 4.90 & 4.73 & 4.73 & 1.07 & & 2,251 & 5.10 & 4.86 & 4.65 & 3.79 & * & 2,188 \\
\hline DV & 4.50 & 4.33 & 4.33 & 1.75 & & 2,252 & 4.44 & 4.65 & 4.07 & 6.83 & $* *$ & 2,188 \\
\hline $\mathrm{KF}$ & 4.55 & 4.43 & 4.45 & 0.95 & & 2,252 & 4.45 & 4.38 & 4.10 & 3.95 & * & 2,188 \\
\hline PLE & 5.06 & 5.03 & 4.68 & 9.20 & $* *$ & 2,252 & 4.88 & 4.84 & 4.52 & 4.10 & * & 2,188 \\
\hline
\end{tabular}

Legende: Prim.: Primarstufe, Sek I: Sekundarstufe I, I: unteres Terzil, II: mittleres Terzil, III: oberes Terzil, LM: Leistungen Mathematik, LD: Leistungen Deutsch, RLM: Relative Leistungen Mathematik, RLD: Relative Leistungen Deutsch, NM: Note Mathematik, ND: Note Deutsch, OF: Offenheit, DV: Durchsetzungsvermögen, KF: Konfliktfähigkeit, PLE: Positive Lebenseinstellung, ${ }^{*}: p<.05,{ }^{* *}: p<.01$

In den Klassen, die im 6. Schuljahr am Ende der Primarstufe standen, waren folgende Unterschiede zu beobachten (Tabelle 1): Wie in Hypothese 1 formuliert, wiesen Schülerinnen und Schüler, die auf eine höhere Akzeptanz bei ihren Klassenkameradinnen und kameraden stiessen, bessere Noten im Fach Deutsch auf. Der Hypothese 2 entsprechend zeigte sich, dass Schülerinnen und Schüler, die auf eine höhere Ablehnung stiessen, schlechtere Noten in den Fächern Deutsch und Mathematik aufwiesen. Sie schnitten auch im Leistungstest Deutsch im klassen- 
internen Vergleich schlechter ab. Sie zeigten zudem schwächere Testleistungen im Fach Deutsch. Der Hypothese 3 entsprechend beschrieben sich besser akzeptierte Schülerinnen und Schüler als durchsetzungsfähiger und berichteten über eine positivere Lebenseinstellung. Der Hypothese 4 entsprechend hatten stärker abgelehnte Schülerinnen und Schüler eine weniger positive Lebenseinstellung. Auch wenn die Mittelwertsunterschiede nicht bei allen untersuchten Variablen signifikant waren, unterschieden sich die Schülerinnen und Schüler der Terzile grösstenteils in der erwarteten Richtung.

In den Klassen, die im 6. Schuljahr am Anfang der Sekundarstufe I standen, zeigte sich folgendes Bild: Es waren keine der in Hypothese 1 formulierten Unterschiede in den Leistungen zwischen den drei Terzilen der Akzeptanz zu finden. Bei den Leistungen im Fach Deutsch zeigte sich entgegen der Hypothese 1, dass Schülerinnen und Schüler, die auf eine höhere Akzeptanz stiessen, schlechter abschnitten. Auch die gemäss Hypothese 2 erwarteten Unterschiede wurden nicht gefunden. Schülerinnen und Schüler, die auf eine höhere Ablehnung bei ihren Klassenkameradinnen stiessen, wiesen im klasseninternen Vergleich bessere Leistungen im Fach Deutsch auf. Die Hypothese 3 wurde ebenfalls widerlegt. Schülerinnen und Schüler, die unterschiedlich akzeptiert wurden, unterschieden sich weder im sozialen Selbstkonzept noch in der positiven Lebenseinstellung voneinander. Entsprechend der Hypothese 4 beschrieben sich stärker abgelehnte Schülerinnen und Schüler als weniger offen, weniger konfliktfähig und wiesen eine weniger positive Lebenseinstellung auf.

Zusammenfassend können die Hypothesen 1 bis 4 für die Gruppe der Schülerinnen und Schüler, die im 6. Schuljahr am Ende der Primarstufe stehen, weitgehend bestätigt werden: Beliebte Schülerinnen und Schüler erbringen im klasseninternen Vergleich bessere Leistungen und Noten und berichten über ein höheres soziales Selbstkonzept. Für die Gruppe, die am Anfang der Sekundarstufe I steht, treffen die Hypothesen 1 bis 3 aber nicht zu. Nur Hypothese 4 wird bestätigt.

\subsection{Stabilität und Veränderung}

$\mathrm{X}^{2}$ Tests dienten der Überprüfung der Hypothesen 5 und 6 zur Stabilität und Veränderung von Akzeptanz und Ablehnung. In einer ersten Analyse wurde die beobachtete Verteilung von Abstieg, Verbleib und Aufstieg vom 5. zum 6. und vom 6. zum 7. Schuljahr einer Gleichverteilung (erwartete Werte) gegenübergestellt. Abstieg, Verbleib und Aufstieg beziehen sich auf Veränderungen in der Zugehörigkeit zu den Terzilen. Aufstieg bezeichnet einen Wechsel von einem Terzil mit weniger positiven Wahlen zu einem mit mehr Wahlen (Terzil I zu II/III oder II zu III). 
Abstieg ist der Wechsel von einem Terzil mit mehr Wahlen zu einem mit weniger Wahlen (Terzil III zu II/I oder Terzil II zu I). Stabil meint, dass die Schülerin oder der Schüler im gleichen Terzil geblieben ist.

Tab. 2 Abstieg, Verbleib und Aufstieg über die Zeit

\begin{tabular}{|c|c|c|c|c|c|c|c|}
\hline & & & $h_{b}\left(h_{e}\right)$ & & & & \\
\hline & & & Abstieg & Verbleib & Aufstieg & $\chi^{2}, d f$ & $p$ \\
\hline \multirow{4}{*}{ 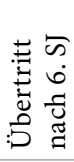 } & \multirow[t]{2}{*}{ Akzeptanz } & 5.-6. SJ (Prim) & $71(85)$ & 124(85) & $60(85)$ & $27.55,2$ & $<.01$ \\
\hline & & 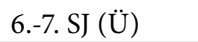 & $82(85)$ & $98(85)$ & $75(85)$ & $3.27,2$ & .20 \\
\hline & \multirow[t]{2}{*}{ Ablehnung } & 5.-6. SJ (Prim.) & $41(85)$ & $150(85)$ & $64(85)$ & $77.67,2$ & $<.01$ \\
\hline & & 6.-7. SJ (Ü) & $58(85)$ & 155(85) & $42(85)$ & $87.98,2$ & $<.01$ \\
\hline \multirow{4}{*}{ 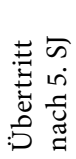 } & \multirow[t]{2}{*}{ Akzeptanz } & 5.-6. SJ (Ü.) & $55(63.7)$ & $79(63.7)$ & $57(63.7)$ & $5.57,2$ & .06 \\
\hline & & 6.-7. SJ (Sek I) & $39(63.7)$ & $115(63.7)$ & $37(63.7)$ & $62.12,2$ & $<.01$ \\
\hline & \multirow[t]{2}{*}{ Ablehnung } & 5.-6. SJ (Ü.) & $27(63.7)$ & $107(63.7)$ & $57(63.7)$ & $51.31,2$ & $<.01$ \\
\hline & & 6.-7. SJ (Sek I) & $45(63.7)$ & $120(63.7)$ & $26(63.7)$ & $77.60,2$ & $<.01$ \\
\hline
\end{tabular}

Legende: SJ: Schuljahr, Prim.: Verbleib in der Primarstufe I, Ü: Übertritt in die Sekundarstufe I, Sek I.: Verbleib in der Sekundarstufe I

Bei der Gruppe mit Übertritt nach dem 6. Schuljahr waren entsprechend Hypothese 5 vom 5. zum 6. Schuljahr beim Verbleib in der Primarstufe die Akzeptanz und die Ablehnung stabiler als bei einer Gleichverteilung (Tabelle 2). Entgegen Hypothese 6 war bei der Ablehnung die Häufigkeit von Verbleib beim Übergang vom 6. zum 7. Schuljahr mit dem Übertritt in die Sekundarstufe I häufiger als erwartet. Bei der Akzeptanz wurden der Hypothese 6 entsprechend keine Abweichungen von der Gleichverteilung gefunden.

Bei der Gruppe mit Übertritt nach dem 5. Schuljahr war vom 5. zum 6. Schuljahr mit Übertritt in die Sekundarstufe I bei der Ablehnung entgegen Hypothese 6 die Zahl der Personen im Verbleib höher als erwartet. Der Hypothese 5 entsprechend waren vom 6. zum 7. Schuljahr beim Verbleib in der Sekundarstufe I signifikante Abweichungen bei Akzeptanz und Ablehnung vorhanden. Insgesamt bestätigen diese Auswertungen die Hypothesen 5 und 6 weitgehend. Sowohl die Akzeptanz als auch die Ablehnung bleiben über die Zeit relativ stabiler. Findet der Übertritt in die Sekundartstufe I mit einem Klassenwechsel statt, ist die Stabilität der Akzeptanz geringer.

Aufgrund der berichteten hypothesenwidrigen Befunde wurden die Hypothesen 5 und 6 zusätzlich mit einer alternativen Vorgehensweise überprüft. Dazu wurden die beobachteten Häufigkeiten von Abstieg, Verbleib und Aufstieg vom 
5. zum 6. Schuljahr als Erwartungswerte $\left(h_{e}\right)$ eingesetzt und mit den beobachteten Häufigkeiten von Abstieg, Verbleib und Aufstieg vom 6. zum 7. Schuljahr $\left(h_{b}\right)$ verglichen. Damit konnte überprüft werden, ob die Verteilung der Werte vom 5. zum 6. Schuljahr mit der Verteilung vom 6. zum 7. Schuljahr übereinstimmt. Bei der Gruppe mit Übertritt nach dem 6. Schuljahr stammen die erwarteten Häufigkeiten aus der Phase ohne Übertritt und die beobachteten Häufigkeiten aus der Phase mit Übertritt. Bei der Gruppe mit Übertritt nach dem 5. Schuljahr sind die erwarteten Häufigkeiten aus der Phase mit Übertritt und die beobachten Häufigkeiten aus der Phase ohne Übertritt. Diese direkte Gegenüberstellung ist möglich, weil die Randsummen zur Berechnung der Erwartungswerte mit den Randsummen der beobachteten Werte übereinstimmen.

Tab. 3 Vergleiche der Stabilität mit und ohne Übertritt

\begin{tabular}{llrrrrr}
\hline & & $\begin{array}{c}\text { Abstieg } \\
\mathrm{h}_{\mathrm{b}}\left(\mathrm{h}_{\mathrm{e}}\right)\end{array}$ & $\begin{array}{c}\text { Verbleib } \\
\mathrm{h}_{\mathrm{b}}\left(\mathrm{h}_{\mathrm{e}}\right)\end{array}$ & $\begin{array}{c}\text { Aufstieg } \\
\mathrm{h}_{\mathrm{b}}\left(\mathrm{h}_{\mathrm{e}}\right)\end{array}$ & $\chi^{2}, d f$ & $p$ \\
\hline Übertritt nach 6. SJ & Akzeptanz & $\mathbf{8 2}(71)$ & $\mathbf{9 8}(124)$ & $\mathbf{7 5}(60)$ & $10.91,2$ & $<.01$ \\
\cline { 2 - 7 } & Ablehnung & $\mathbf{5 8}(41)$ & $\mathbf{1 5 5}(150)$ & $\mathbf{4 2}(64)$ & $14.78,2$ & $<.01$ \\
\hline Übertritt nach 5. SJ & Akzeptanz & $39(\mathbf{5 5})$ & $115(\mathbf{7 9})$ & $37(\mathbf{5 7})$ & $28.08,2$ & $<.01$ \\
\cline { 2 - 7 } & Ablehnung & $45(\mathbf{2 7})$ & $120(\mathbf{1 0 7})$ & $26(57)$ & $30.44,2$ & $<.01$ \\
\hline
\end{tabular}

Legende: fett: Häufigkeiten in einer Phase mit Übertritt in die Sekundarstufe I, SJ: Schuljahr

Dieser Vergleich zeigt gemäss Tabelle 3, dass beim Übertritt in die Sekundarstufe I Abstiege und Aufstiege bei der Akzeptanz häufiger und Verbleibe seltener auftreten, als beim Verbleib in der Primarstufe. Während z. B. bei der Gruppe mit Übertritt nach dem 6. Schuljahr vom 5. zum 6. Schuljahr 124 Schülerinnen und Schüler in ihrer Akzeptanz stabil blieben, waren es ein Jahr später mit Übertritt nur 98. Bei der Gruppe mit Übertritt nach dem 5. Schuljahr blieben vom 5. zum 6. Schuljahr während des Übertritts nur 79 stabil, ein Jahr später ohne Übertritt hingegen 115.

Bei der Ablehnung ist die Stabilität nur bei der Gruppe mit Übertritt nach dem 5. Schuljahr geringer, wenn der Übertritt stattfand (vom 5. zum 6. Schuljahr: 107), als wenn keiner stattfand (vom 6. zum 7. Schuljahr: 120). Möglicherweise verfestigt sich die Ablehnung gegenüber Schülerinnen und Schülern über die Zeit immer mehr. Dies hat zur Folge, dass bei späterem Übertritt in die Sekundarstufe I weniger Auswirkungen auf die Stabilität der Ablehnung zu finden sind.

Insgesamt belegen diese Auswertungen die hohe Stabilität der Beliebtheit (Akzeptanz und Ablehnung) über ein Schuljahr (Hypothese 5). Die vorgenommenen Auswertungen bestätigen die Hypothese 6, die besagt, dass Veränderungen der 
Beliebtheit beim Übertritt in die Sekundarstufe I häufiger auftreten als beim Verbleib in der gleichen Klasse. Dies trifft stärker für die Akzeptanz als für die Ablehnung zu.

\subsection{Determinanten der Veränderung}

Weil die Veränderungen der Beliebtheit beim Übergang in die Sekundarstufe I besonders häufig sind, wurden mit der Teilstichprobe mit Übertritt nach dem 6. Schuljahr die Hypothesen 7 bis 10 getestet. Der Einfluss des zugewiesenen Schulniveaus der Sekundarstufe I und der Leistungsposition innerhalb der neuen Klasse auf die Veränderung der Beliebtheit wurde geprüft. Dieses Vorgehen ermöglichte es, Einblicke in die Entstehung der Beliebtheit zu gewinnen.

Die Testung der Hypothesen erfolgte mittels logistischer Regressionen. Als abhängige Variable dienten die Veränderungen der Akzeptanz und der Ablehnung vom 6. zum 7. Schuljahr (Abstieg vs. Aufstieg). Die Schülerinnen und Schüler, die in dieser Zeit stabil blieben, wurden nicht in die Analyse aufgenommen. Als unabhängige Variablen wurden das dummy-kodierte Schulniveau (Grundanforderung und hohe Anforderungen) und die relativen Mathematikleistungen herangezogen.

Tab. 4 Determinanten der Veränderung beim Übertritt nach dem 6. Schuljahr (odds ratios)

\begin{tabular}{|c|c|c|c|c|c|}
\hline \multirow{3}{*}{ Tiefe Anf. } & \multicolumn{2}{|c|}{ Akzeptanz } & \multicolumn{3}{|c|}{ Ablehnung } \\
\hline & $\operatorname{Exp}(B)$ & CI $95 \%$ & \multirow{2}{*}{$\frac{\operatorname{Exp}(\mathrm{B})}{.38}$} & \multicolumn{2}{|c|}{ CI $95 \%$} \\
\hline & $2.75 *$ & 1.096 .93 & & .11 & 1.24 \\
\hline Hohe Anf. & .94 & $.45 \quad 1.98$ & $2.64 *$ & 1.01 & 6.88 \\
\hline RLM & $1.73 *$ & $1.06 \quad 2.83$ & .63 & .33 & 1.22 \\
\hline \multirow[t]{2}{*}{ Konstante } & .79 & & .63 & & \\
\hline & $\begin{array}{l}r^{2} \text { (Nagelkerke } \\
p<.01, N=154\end{array}$ & $10.58, d f=3$, & $\begin{array}{l}r^{2} \text { (Nagelkerk } \\
p<.01, N=98\end{array}$ & $x^{2}=1$ & $03, d f=3$ \\
\hline
\end{tabular}

Legende: Anf.: Anforderungen Sek I Niveau, RLM: Relative Leistungen Mathematik, ${ }^{*}: p<.05$

Den Hypothesen 7 und 8 entsprechend beeinflusste das Schulniveau auf der Sekundarstufe I die Veränderung der Akzeptanz und der Ablehnung. Die Wahrscheinlichkeit eines Aufstiegs in der Akzeptanz war im Schulniveau mit Grundanforderungen 2.75-mal höher als im Schulniveau mit mittleren Anforderungen. Die Wahrscheinlichkeit, nach dem Übertritt stärker abgelehnt zu werden, war 
demgegenüber im Schulniveau mit hohen Anforderungen 2.64-mal höher als im Schulniveau mit mittleren Anforderungen.

Entsprechend der Hypothese 9 wiesen die Mathematikleistungen im Klassenvergleich einen positiven Effekt auf die Veränderung der Akzeptanz auf. Ihr Effekt auf die Veränderung der Ablehnung war entgegen der Hypothese 10 nicht signifikant.

\section{Diskussion}

Der vorliegende Beitrag befasst sich mit der Beliebtheit von Schülerinnen und Schülern anhand der zwei Dimensionen Akzeptanz und Ablehnung. Es wurde erstmals gezeigt, welche Konsequenzen der Übertritt in die leistungssegregierte Sekundarstufe I auf die Beliebtheit hat. Dabei wurde auf den Einfluss von individuellen und Klassenleistungen fokussiert. Die durchgeführten Analysen belegen, dass der Übertritt in die Sekundarstufe I mit Veränderungen der Beliebtheit einhergehen, die mit dem Big-Fish-Little-Pond-Effekt (Marsh, 1987, 2005) vergleichbar sind. Die Beliebtheit der Kinder verändert sich beim Schulübertritt abhängig vom zugewiesenen Schulniveau und der Leistungsposition in der Klasse in Mathematik.

Die Befunde zur Validierung von Akzeptanz und Ablehnung am Ende der Primarstufe bestätigen bekannte Unterschiede der Beliebtheit von Schülerinnen und Schülern, die sich in ihren Leistungen voneinander unterscheiden. Es sind insbesondere Schülerinnen und Schüler mit geringeren Schulleistungen, welche abgelehnt werden. Am Anfang der Sekundarstufe I sind solche leistungsbezogenen Differenzen hingegen noch nicht zu finden. Schülerinnen und Schüler mit geringeren Leistungen sind dann eher beliebter und solche mit besseren Leistungen werden eher abgelehnt. Wir vermuten, dass unmittelbar nach dem Übertritt in die gegliederte Sekundarstufe I schulische Leistungen durch veränderte Vergleichsprozesse in einem neuen Licht erscheinen.

Auch die Analysen zur Stabilität von Akzeptanz und Ablehnung legen nahe, dass die Validierung in der Situation zu Beginn der Sekundarstufe I nicht gelingt, weil nach dem Übertritt soziale Positionen in den Klassen neu ausgehandelt werden. Die Beliebtheit von Schülerinnen und Schülern verändert sich beim Übertritt, der mit einem Wechsel der Bezugsgruppe einhergeht, stärker als beim Verbleib in der gleichen Klasse.

Die durchgeführten logistischen Regressionen lassen vermuten, dass zumindest ein Teil dieser Veränderungen auf Ergebnisse von Leistungsvergleichen innerhalb der neu zusammengesetzten Klassen zurückzuführen ist. Weil individuelle Leistungen je nach Vergleichsgruppe unterschiedlich beurteilt werden, sind Schülerinnen 
und Schüler, welche in eine Klasse mit höheren Anforderungen wechseln, in der neuen Klasse weniger attraktiv als vor dem Übertritt. Sie verlieren an Beliebtheit. Andererseits profitieren Schülerinnen und Schüler, die im Klassenvergleich bessere Leistungen zeigen, vom Übertritt. Sie gewinnen an Beliebtheit.

Weiter konnte gezeigt werden, dass sich beliebtere Schülerinnen und Schüler am Ende der Primarstufe als durchsetzungsfähiger einschätzen und über eine positivere Lebenseinstellung verfügen. Zu Beginn der Sekundarstufe I korrespondieren ein geringeres Durchsetzungsvermögen, weniger Offenheit und Konfliktfähigkeit sowie eine weniger positive Lebenseinstellung mit einer stärkeren Ablehnung. Möglicherweise stellt die Situation nach dem Übertritt eine sensible Phase für Schülerinnen und Schüler dar, in welcher soziale Selbstkonzepte besonders stark mit Ablehnung durch andere interagieren.

Aufgrund der hohen Ausfallquoten beim Schulübergang und der fehlenden Beachtung der geschachtelten Mehrebenenstruktur der Stichprobe sollten die Ergebnisse vor einer weitergehenden Interpretation repliziert werden. Weil die Veränderung der Beliebtheit bei Schulübertritt bisher kaum untersucht worden ist, erachten wir bereits die deskriptiven Vergleiche am Ende der Primarstufe und zu Beginn der Sekundarstufe I als interessant. Zusätzlich liefern die weiteren Ergebnisse Erklärungen, wie sich die Beliebtheit in Abhängigkeit vom zugewiesenen Schulniveau und der Leistungsposition in Mathematik verändert. Schulleistungen stellen aber nur eines von vielen Merkmalen dar, welches die Beliebtheit beeinflusst. Es sind Untersuchungen wünschenswert, die den Einfluss weiterer Merkmale von Schülerinnen und Schülern, Schulklassen und Lehrpersonen auf die Veränderung der Beliebtheit beim Schulübertritt einbeziehen. Ebenso wichtig ist die Umsetzung langfristig angelegter Studien, welche die Folgen der Veränderung der Beliebtheit beim Schulübertritt auf die psychosoziale Entwicklung der Jugendlichen untersuchen. Damit kann unser Verständnis der Prozesse, die beim Schulübertritt ablaufen, vertieft werden.

\section{Literatur}

Allen, J. P., Porter, M. R., McFarland, F. C., Marsh, P., \& McElhaney, K. B. (2005). The two faces of adolescents' success with peers: Adolescent popularity, social adaptation, and deviant behavior. Child Development, 76(3), 747-760.

Angelone, D., Keller, F., \& Moser, U. (2013). Entwicklung schulischer Leistungen während der obligatorischen Schulzeit. Bericht zur vierten Zürcher Lernstandserhebung zuhanden der Bildungsdirektion des Kantons Zürich. Zürich: Bildungsdirektion Kanton Zürich. 
Attili, G., Vermigli, P., \& Roazzi, A. (2010). Children's social competence, peer status, and the quality of mother-child and father-child relationships. European Psychologist, 15(1), 23-33. doi: 10.1027/1016-9040/a000002

Babad, E. (2001). On the conception and measurement of popularity: more facts and some straight conclusions. Social Psychology of Education, 5, 3-30.

Cillessen, A. H. N., van Ijzendoorn, H. W., Van Lieshout, C. F. M., \& Hartup, W. W. (1992). Heterogeneity among peer-rejected boys: Subtypes and stabilities. Child Development, 63(4), 893-905.

Dunkake, I. (2012). Soziale Netzwerke von Schülern: Beispiele angewandter Netzwerkanalysen. In M. Hennig \& C. Stegbauer (Hrsg.), Die Integration von Theorie und Methode in der Netzwerkforschung. Wiesbaden: Springer VS.

Grob, A., Lüthi, R., Kaiser, F. G., Flammer, A., Mackinnon, A., \& Wearing, A. J. (1991). Berner Fragebogen zum Wohlbefinden Jugendlicher (BFW). Diagnostica, 37, (66-75).

Haberman, S. J. (2009). Linking parameter estimates derived from an item response model through separate calibrations. ETS Research Report Series. Princeton, NJ: ETS.

Hannover, B., \& Kessels, U. (2009). Gleichaltrige. In E. Wild \& J. Möller (Hrsg.), Pädagogische Psychologie (S. 283-304). Berlin, Heidelberg: Springer.

Hardy, C. L., Bukowski, W. M., \& Sippola, L. K. (2002). Stability and change in peer relationships during the transition to middle-level school. Journal of Early Adolescence, 22(2), 117-142.

Hatzichristou, C., \& Hopf, D. (1996). A multiperspective comparison of peer sociometric status groups in childhood and adolescence. Child Development, 67(3), 1085-1102. doi: $10.2307 / 1131881$

Jiang, X. L., \& Cillessen, A. H. N. (2005). Stability of continuous measures of sociometric status: a meta-analysis. Developmental Review, 25(1), 1-25. doi: 10.1016/j.dr.2004.08.008

Lansford, J. E., Killeya-Jones, L. A., Miller, S., \& Costanzo, P. R. (2009). Early adolescents' social standing in peer groups: behavioral correlates of stability and change. Journal of Youth and Adolescence, 38(8), 1084-1095. doi: 10.1007/s10964-009-9410-3

Marsh, H. W. (1987). The Big-Fish-Little-Pond effect on academic self-concept. Journal of Educational Psychology, 79(3), 280-295.

Marsh, H. W. (2005). Big-Fish-Little-Pond Effect on academic self-concept. Zeitschrift für Pädagogische Psychologie, 19(3), 119-129. doi: 10.1024/1010-0652.19.3.119

Moreno, J. L. (1978). Who Shall Survive? Foundations of sociometry, group psychotherapy and sociodrama (3 ed.). Beacon, New York: Beacon House Inc.

Moreno, J. L. (Hrsg.). (1960). The sociometry reader. Glencoe, IL: The Free Press.

Moser, U., Buff, A., Angelone, D., \& Hollenweger, J. (2011). Nach sechs Jahren Primarschule. Deutsch, Mathematik und motivational-emotionales Befinden am Ende der 6. Klasse. Zürich: Bildungsdirektion Kanton Zürich.

Neuenschwander, M. P. \& Hascher, T. (2003). Zufriedenheit von Schülerinnen und Schülern und ihre soziale Integration. Psychologie in Erziehung und Unterricht, 50, 270-280.

Neuenschwander, M. P., Rösselet, S., Niederbacher, E., Rottermann, B., \& Scheffler, L. (2014). Wirkungen der Selektion WiSel. Dokumentation des Fragebogens für Schülerinnen und Schüler der Welle 3 2013/2014 (Forschungsbericht). Solothurn: Pädagogische Hochschule FHNW, Zentrum Lernen und Sozialisation.

Neuenschwander, M. P., Rösselet, S., Rottermann, B., Singer, A., Scheffler, L., \& Ziehli, M. (2013). Wirkungen der Selektion WiSel. Dokumentation des Fragebogens für Schülerinnen 
und Schüler der Welle 1 2011/2012 (Forschungsbericht). Solothurn: Pädagogische Hochschule FHNW, Zentrum Lernen und Sozialisation.

Neuenschwander, M. P., Rösselet, S., Scheffler, L., \& Rottermann, B. (2014). Wirkungen der Selektion WiSel. Dokumentation des Fragebogens für Schülerinnen und Schüler der Welle 22012 (Forschungsbericht). Solothurn: Pädagogische Hochschule FHNW, Zentrum Lernen und Sozialisation.

Neuenschwander, M. P., Rottermann, B., Niederbacher, E., Rösselet, S., \& Scheffler, L. (2014). Wirkungen der Selektion WiSel. Dokumentation der Leistungstests Mathematik und Deutsch der Welle 3 2013/2014 (Forschungsbericht). Solothurn: Pädagogische Hochschule FHNW, Zentrum Lernen und Sozialisation.

Neuenschwander, M. P., Rottermann, B., \& Rösselet, S. (2013). Wirkungen der Selektion WiSel. Dokumentation der Leistungstests Mathematik und Deutsch der Welle 1 2011/2012 (Forschungsbericht). Solothurn: Pädagogische Hochschule FHNW, Zentrum Lernen und Sozialisation.

Neuenschwander, M. P., Rottermann, B., Scheffler, L., \& Rösselet, S. (2014). Wirkungen der Selektion WiSel. Dokumentation der Leistungstests Deutsch und Mathematik der Welle 22012 (Forschungsbericht). Solothurn: Pädagogische Hochschule FHNW, Zentrum Lernen und Sozialisation.

Newcomb, A. F., Bukowski, W. M., \& Pattee, L. (1993). Children's peer relations: A meta-analytic review of popular, rejected, neglected, controversial, and average sociometric status. Psychological Bulletin, 113(1), 99-128. doi: 10.1037/0033-2909.113.1.99

Ollendick, T. H., Weist, M. D., Borden, M. C., \& Greene, R. W. (1992). Sociometric status and academic, behavioral, and psychological adjustment: A five-year longitudinal study. Journal of Consultingand Clinical Psychology, 60(1), 80-87.

Parkhurst, J. T., \& Asher, S. R. (1992). Peer rejection in middle school: subgroup differences in behavior, loneliness, and interpersonal concerns. Developmental Psychology, 28(2), 231-241.

Pellegrini, A. D., \& Bartini, M. (2000). A Longitudinal study of bullying, victimization, and peer affiliation during the transition from Primary school to middle school. American Educational Research Journal, 37(3), 699-725.

Rost, D. H., \& Czeschlik, T. (1994). Beliebt und intelligent? Abgelehnt und Dumm? Eine soziometrische Studie an 6500 Grundschulkindern. Zeitschrift für Sozialpsychologie, 25, 170-176.

Rubin, K. H., Bukowski, W. M., \& Parker, J. G. (2006). Peer interactions, relationships, and groups. In N. Eisenberg (Ed.), Handbook of child psychology: Social, emotional, and personality development (pp. 571-645). New York: Wiley.

Tamm, A., Kasearu, K., \& Tulviste, T. (2014). The role of family in adolescents' peer acceptance. Personal Relationships, 21(3), 420-432. doi: 10.1111/pere.12041

Thibaut, J. W. \& Kelley, H. H. (1959). The social psychology of groups. New York: Wiley.

Trautwein, U., \& Baeriswyl, F. (2007). Wenn leistungsstarke Klassenkameraden ein Nachteil sind. Zeitschrift für Pädagogische Psychologie, 21(2), 119-133. doi: 10.1024/10100652.21.2.119

van der Linden, D., Scholte, R. H. J., Cillessen, A. H. N., Nijenhuis, J. T., \& Segers, E. (2010). Classroom ratings of likeability and popularity are related to the Big Five and the general factor of personality. Journal of Research in Personality, 44(5), 669-672. doi: 10.1016/j. jrp.2010.08.007 
Walker, S. (2009). Sociometric stability and the behavioral correlates of peer acceptance in early childhood. The Journal of Genetic Psychology, 170(4), 339-358. doi: 10.1080/00221320903218364

Warm, T. A. (1989). Weighted likelihood estimation of ability in item response theory. Psychometrika, 54(3), 427-450.

Wentzel, K. R. (2005). Peer relationships, motivation, and academic performance at school. In A. J. Elliot \& C. S. Dweck (Eds.), Handbook of competence and motivation (pp. 279296). New York: Guilford Publications.

Yen, W. M., \& Fitzpatrick, A. R. (2006). Item response theory. In R. L. Brennan (Ed.), Educational Measurement (pp. 111-154). Westport: Praeger Publishers.

Open Access Dieses Kapitel wird unter der Creative Commons Namensnennung 4.0 International Lizenz (http://creativecommons.org/licenses/by/4.0/deed.de) veröffentlicht, welche die Nutzung, Vervielfältigung, Bearbeitung, Verbreitung und Wiedergabe in jeglichem Medium und Format erlaubt, sofern Sie den/die ursprünglichen Autor(en) und die Quelle ordnungsgemäß nennen, einen Link zur Creative Commons Lizenz beifügen und angeben, ob Änderungen vorgenommen wurden.

Die in diesem Kapitel enthaltenen Bilder und sonstiges Drittmaterial unterliegen ebenfalls der genannten Creative Commons Lizenz, sofern sich aus der Abbildungslegende nichts anderes ergibt. Sofern das betreffende Material nicht unter der genannten Creative Commons Lizenz steht und die betreffende Handlung nicht nach gesetzlichen Vorschriften erlaubt ist, ist für die oben aufgeführten Weiterverwendungen des Materials die Einwilligung des jeweiligen Rechteinhabers einzuholen.

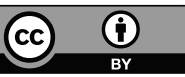

